\title{
Stability and formation of the resonant system HD 73526
}

\author{
Zs. Sándor ${ }^{1,2}$, W. Kley ${ }^{2}$, and P. Klagyivik ${ }^{1}$ \\ 1 Department of Astronomy, Eötvös Loránd University, Pázmány Péter sétány 1/A, 1117 Budapest, Hungary \\ e-mail: [Zs.Sandor;P.Klagyivik]@astro.elte.hu \\ 2 Institut für Astronomie und Astrophysik, Abt. Computational Physics, Universität Tübingen, Auf der Morgenstelle 10, \\ 72076 Tübingen, Germany \\ e-mail: kley@tat.physik.uni-tuebingen.de
}

Received 23 February 2007 / Accepted 4 June 2007

ABSTRACT

\begin{abstract}
Context. Based on radial velocity measurements, it has been found that the two giant planets detected around the star HD 73526 are in 2:1 resonance. However, as our numerical integration shows, the derived orbital data for this system result in chaotic behavior of the giant planets, which is uncommon among the resonant extrasolar planetary systems.

Aims. We present regular (non-chaotic) orbital solutions for the giant planets in the system HD 73526 and offer formation scenarios based on combining planetary migration and sudden perturbative effects such as planet-planet scattering or rapid dispersal of the protoplanetary disk. A comparison with the already-studied resonant system HD 128311, exhibiting similar behavior, is given.

Methods. The new sets of orbital solutions were derived using the Systemic Console. The stability of these solutions was investigated using the Relative Lyapunov indicator, while the migration and scattering effects are studied by gravitational $N$-body simulations applying non-conservative forces. Additionally, hydrodynamic simulations of embedded planets in protoplanetary disks were performed to follow the capture into resonance.

Results. For the system HD 73526 we demonstrate that the observational radial velocity data are consistent with a coplanar planetary system in a stable 2:1 resonance exhibiting apsidal corotation. We have shown that, similarly to the system HD 128311, the present dynamical state of HD 73526 could be the result of a mixed evolutionary process combining planetary migration and a perturbative event.
\end{abstract}

Key words. planets and satellites: formation - celestial mechanics - hydrodynamics - methods: $N$-body simulations

\section{Introduction}

Nearly one third of multiplanet extrasolar planetary systems contain pairs of giant planets engulfed in mean motion resonances. Resonant extrasolar planetary systems have special importance when studying the formation of extrasolar planetary systems as they require a dissipative mechanism operating on the planets that is capable of changing the semi-major axis of their orbits. Hence, resonant planetary systems may support the planetary migration scenario, and thus help in answering the question why Jupiter-sized giant planets do not orbit at their place of formation. According to the classical formation theories such as planetesimal accretion followed by core instability or the gravitational instability model for giant planets, it is generally accepted that they formed quite far from their host star (beyond the snowline, which is $a \sim 3-4$ AU for a Sun-like star), where volatile elements can condense and accrete (e.g. Hayashi 1981; Pollack 1984; Perryman 2000). On the other hand, observations show that a large fraction of Jupiter-sized planets are found very close to their host star at distances smaller than $a=1 \mathrm{AU}$.

Planetary migration occurs when the planets are still embedded in a protoplanetary disk and, due to tidal interactions between the planets and the disk, their semi-major axes decrease. During the migration process, a resonant capture can occur between two planets, if certain dynamical conditions are fulfilled. Once the capture happens and the orbital decay is sufficiently slow (adiabatic), the resonant configuration is preserved during the migration, and the two planets can travel very close to their host star. The efficiency of this mechanism is clearly demonstrated by the well-known resonant system around the star GJ 876. The giant planets in this system are engaged in a 2:1 resonance, having very well-determined elements due to their short orbital periods ( $P_{1} \approx 30, P_{2} \approx 60$ days). It is true in general cases (Beaugé et al. 2006) and also in the particular case of GJ 876 (Lee \& Peale 2002; Kley et al. 2005) that as a result of an adiabatic convergent migration process, beside the mean motion resonance, the orbits of the giant planets also exhibit apsidal corotation (or in other words apsidal resonance) where the osculating orbital ellipses of the giant planets rotate with the same mean angular velocity.

Numerical integrations based on observed orbital data of the planetary system HD 128311 (Vogt et al. 2005) show that the giant planets are in a 2:1 mean motion resonance without exhibiting apsidal corotation. Sándor \& Kley (2006) offered a mixed evolutionary scenario for this system combining an adiabatic migration leading the system into a $2: 1$ resonance and a sudden perturbation. This perturbation could be either a close encounter between one of the giants and a relatively small mass $\left(\sim 10 M_{\oplus}\right)$ planet already existing in the system (which we will refer to in the following as planet-planet scattering), or the fast termination of the planetary migration due to a sudden dispersal of the protoplanetary disk. Both these perturbations can be strong enough to induce relatively large-amplitude oscillations of the eccentricities and the resonant angles. While retaining the mean motion resonance, the planet-planet scattering may even break the apsidal corotation between the orbits of the giant planets. 
In this paper we will support the above mixed evolutionary scenario by the detailed analysis of the newly discovered resonant system HD 73526. Tinney et al. (2006) reported that the giant planets of HD 73526 are in 2:1 resonance. It has been found that only the resonant angle $\Theta_{1}=2 \lambda_{2}-\lambda_{1}-\varpi_{1}$ librates, while both $\Theta_{2}=2 \lambda_{2}-\lambda_{1}-\varpi_{2}$ and $\Delta \varpi=\varpi_{2}-\varpi_{1}$ circulate. Here, $\lambda_{1,2}$ denote the mean longitude of the inner and outer planet, and $\varpi_{1,2}$ their periastron longitude. However, according to our numerical integration, the recently published orbital data by Tinney et al. (2006), as shown in Table 1, result in a weakly chaotic and irregular behavior of the system.

Since chaotic behavior is uncommon among the known resonant extrasolar planetary systems, and may not guarantee the stability of the giant planets for the whole lifetime of the system, we have searched for regular orbital solutions for the giant planets as well. By using the Systemic Console (http: //www . oklo. org), we have found new coplanar orbital solutions for the giant planets around HD 73526 exhibiting regular behavior. The regular nature (and thus the long-term stability) of these orbits have been checked carefully by numerical integrations and the Relative Lyapunov indicator chaos detection method (Sándor et al. 2004). Numerical integrations based on our newly derived orbital data exhibit very similar behavior of the eccentricities of the planets in HD 73526 to those around HD 128311.

This paper aims at a complete analysis of the resonant system HD 73526 including the stability investigation of the new orbital solutions and modeling the formation of the resonant system HD 73526. The paper is structured as follows: first we present four sets of new orbital data. Then we investigate the stability of these orbital solutions by using the Relative Lyapunov indicator chaos detection method, and finally, we offer possible evolutionary scenarios for the system, which are similar to the studied ones in the case of HD 128311.

\section{Original orbital data and their stability}

It has been mentioned in the case of HD 128311 by Vogt et al. (2005) that a kinematic orbit fit for orbital parameters in the case of a strongly interacting resonant pair of giant planets based only on two unperturbed ellipses often results in a destruction of the system within a few thousand years. This is true for HD 73526 as well.

Tinney et al. (2006) also carried out a self consistent threebody dynamical orbit fit to the observed velocities (as listed in Table 1). They found this dynamical fit to be stable over a $1 \mathrm{Myr}$ integration time. Based on these results we also performed a numerical integration of the system. We found that the giant planets around HD 73526 are indeed in a 2:1 mean motion resonance having libration in the resonance variable $\Theta_{1}$ with an amplitude $\sim 90^{\circ}$.

However, the behavior of the eccentricities is irregular, see Fig. 1. This means that the system is chaotic, which of course may not exclude stability of the giant planets. However, based on the behavior of the observed resonant systems to date, we would prefer orbital solutions exhibiting regular orbits showing a clear dynamical stability for the whole life-time of the system.

By using the Relative Lyapunov indicators (RLI, Sándor et al. 2004), we have mapped the stability properties of the phase space in the close neighborhood of the original orbital data set, in a similar way to that done by Bois et al. (2003) in the case of HD 160691. We have calculated the stability properties of the $a_{1}-a_{2}, e_{1}-e_{2}, M_{1}-M_{2}$, and $\varpi_{1}-\varpi_{2}$ parameter planes, where $a$ is the semi-major axis, $e$ is the eccentricity, $M$ is the mean anomaly and $\varpi$ is the longitude of periastron of one of the giant
Table 1. Original dynamical orbit fit of HD 73526.

\begin{tabular}{cccccc}
\hline \hline Planet & Mass $\left[M_{\mathrm{J}}\right]$ & $a[\mathrm{AU}]$ & $e$ & $M[\mathrm{deg}]$ & $\varpi[\mathrm{deg}]$ \\
\hline inner & 2.9 & 0.66 & 0.19 & 86 & 203 \\
outer & 2.5 & 1.05 & 0.14 & 82 & 13 \\
\hline$\chi_{v}^{2}$ & \multicolumn{2}{c}{$\mathrm{rms}[\mathrm{m} / \mathrm{s}]$} & \multicolumn{3}{c}{ offset velocity $[\mathrm{m} / \mathrm{s}]$} \\
\hline 1.57 & \multicolumn{3}{c}{7.9} & \multicolumn{3}{c}{-29.96} \\
\hline
\end{tabular}

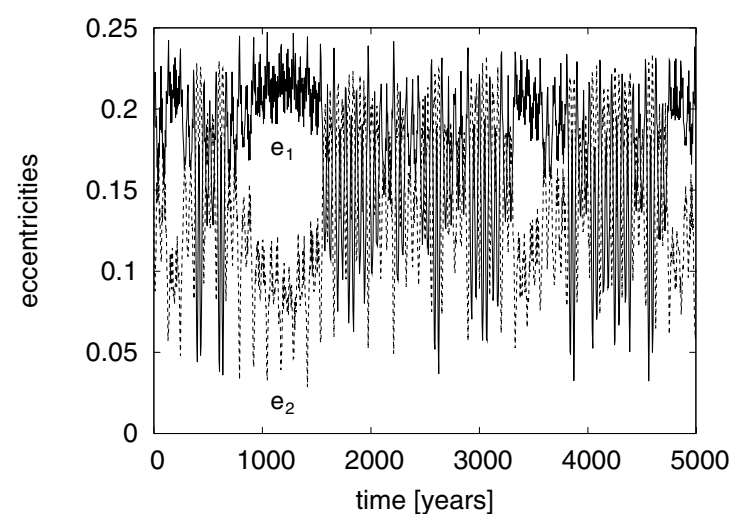

Fig. 1. Irregular behavior of the eccentricities of the giant planets in the system HD 73526 using as initial conditions the data provided by the original dynamical orbit fit (Table 1). The upper (lower) curve shows the behavior of the eccentricity of the inner (outer) planet.

planets, while the indices " 1 " and " 2 " refer to the inner and the outer planet, respectively.

In Fig. 2 we display the stability structures of the parameter planes for the semi-major axis and the eccentricities. During the calculation of a particular parameter plane the other orbital data have been kept fixed. This implies that when investigating the parameter plane of the semi-major axes $a_{1}-a_{2}$, the other orbital data such as the eccentricities $\left(e_{1}, e_{2}\right)$, the mean anomalies $\left(M_{1}, M_{2}\right)$, and the arguments of the periastrons $\left(\varpi_{1}, \varpi_{2}\right)$ have been kept fixed to their original values (Table 1). On each parameter plane we have marked the stable regions white, the weakly chaotic regions grey and the strongly chaotic regions black. The values of the corresponding orbital data are shown with a symbol "+" on each parameter plane. By studying the different stability maps (also the not displayed but calculated cases of the parameter planes $\varpi_{1}-\varpi_{2}$ and $M_{1}-M_{2}$ ), we conclude that the orbital data given by Tinney et al. (2006) are embedded in a weakly chaotic region. We stress that this does not automatically imply the instability of their fit, however by using these orbital data the system exhibits irregular (or chaotic) behavior and may be destabilized at later times.

We have also studied the stability of the system HD 73526 with slightly modified initial orbital elements. We have found, for instance, that even a small error of the initial semi-major axis $\left(\delta a_{2}=0.01\right)$ or eccentricity $\left(\delta e_{2}=0.01\right)$ of the outer planet leads to the destruction of the system in a few hundred thousand years. The above errors $\left(\delta a_{2}\right.$ and $\left.\delta e_{2}\right)$ are well inside the error limits of the original orbit fit of Tinney et al. (2006), being $\Delta a_{2}=$ \pm 0.08 and $\Delta e_{2}= \pm 0.09$. Thus we believe that the use of initial orbital data resulting in regular orbits is more convenient when investigating the system HD 73526.

On the other hand, the possibility that the system shows chaotic behavior as a result of its formation scenario cannot be excluded. It can also happen that a close encounter with a small mass body results in chaotic behavior (characterized by the irregular variation of the eccentricities for instance), though the 

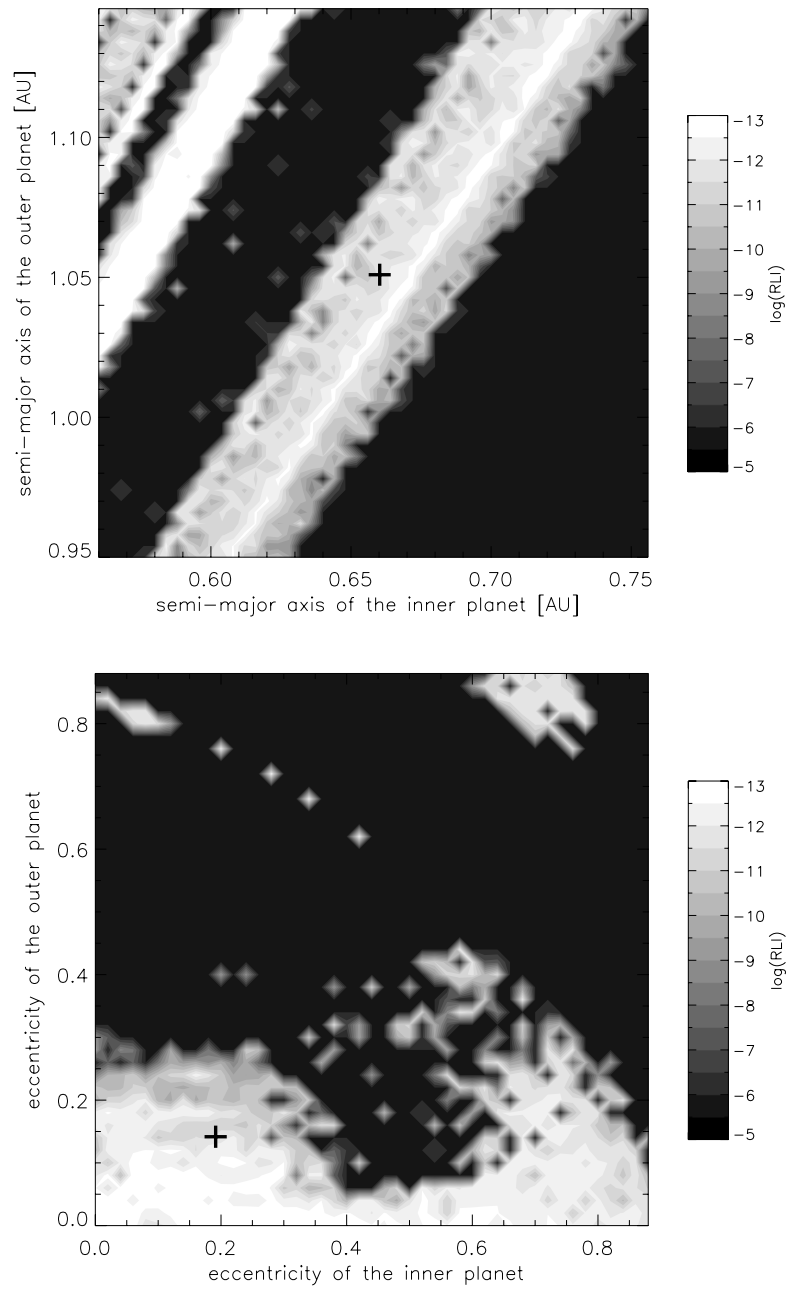

Fig. 2. Stability maps calculated by using the original dynamical orbit fit listed in Table 1. The structure of the parameter planes indicates clearly that the orbital data given by the original fit (marked by "+") are in a weakly chaotic region. Here brighter areas refer to more stable and darker ones to unstable regions.

system itself remains stable for very long time. However, as our numerical simulations show, we have not found such a behavior; either the system shows regular behavior, or it is destroyed soon after the scattering event.

\section{New orbital data and their stability}

To find an orbital solution for the system HD 73526 exhibiting regular behavior, we have used the Systemic Console (http://www . oklo.org), and have found some new orbital fits for the giant panets. In Table 2 we list four sets of them, displaying different behavior. In the case of the first three sets of our orbital data, beside the mean motion resonance, the giant planets are also in apsidal corotation but with enlarged amplitudes in the resonant angles $\Theta_{2}$ or $\Delta \varpi$, while in the fourth case the apsidal corotation is no longer present. In all four cases the eccentricities of the giant planets show relatively large oscillations. The behavior of the eccentricities is very similar to those found in the case of HD 128311 indicating that the present behavior of the system HD 73526 is not likely to be the result of a smooth adiabatic migration scenario alone.
Table 2. New dynamical orbit fits of the giant planets around HD 73526.

\begin{tabular}{ccccccc}
\hline \hline Fit & Planet & Mass $\left[M_{\mathrm{J}}\right]$ & $a[\mathrm{AU}]$ & $e$ & $M[\mathrm{deg}]$ & $\varpi[\mathrm{deg}]$ \\
\hline 1 & inner & 2.42 & 0.66 & 0.26 & 69.8 & 206.6 \\
& outer & 2.58 & 1.045 & 0.16 & 163.2 & 265.6 \\
\hline 2 & inner & 2.62 & 0.66 & 0.209 & 77.7 & 208.5 \\
& outer & 2.56 & 1.047 & 0.194 & 131 & 316.2 \\
\hline 3 & inner & 2.415 & 0.659 & 0.26 & 70.7 & 202.9 \\
& outer & 2.55 & 1.045 & 0.107 & 170.7 & 253.7 \\
\hline 4 & inner & 2.675 & 0.66 & 0.209 & 80.1 & 207.1 \\
& outer & 2.53 & 1.048 & 0.172 & 122.4 & 327.6 \\
\hline
\end{tabular}
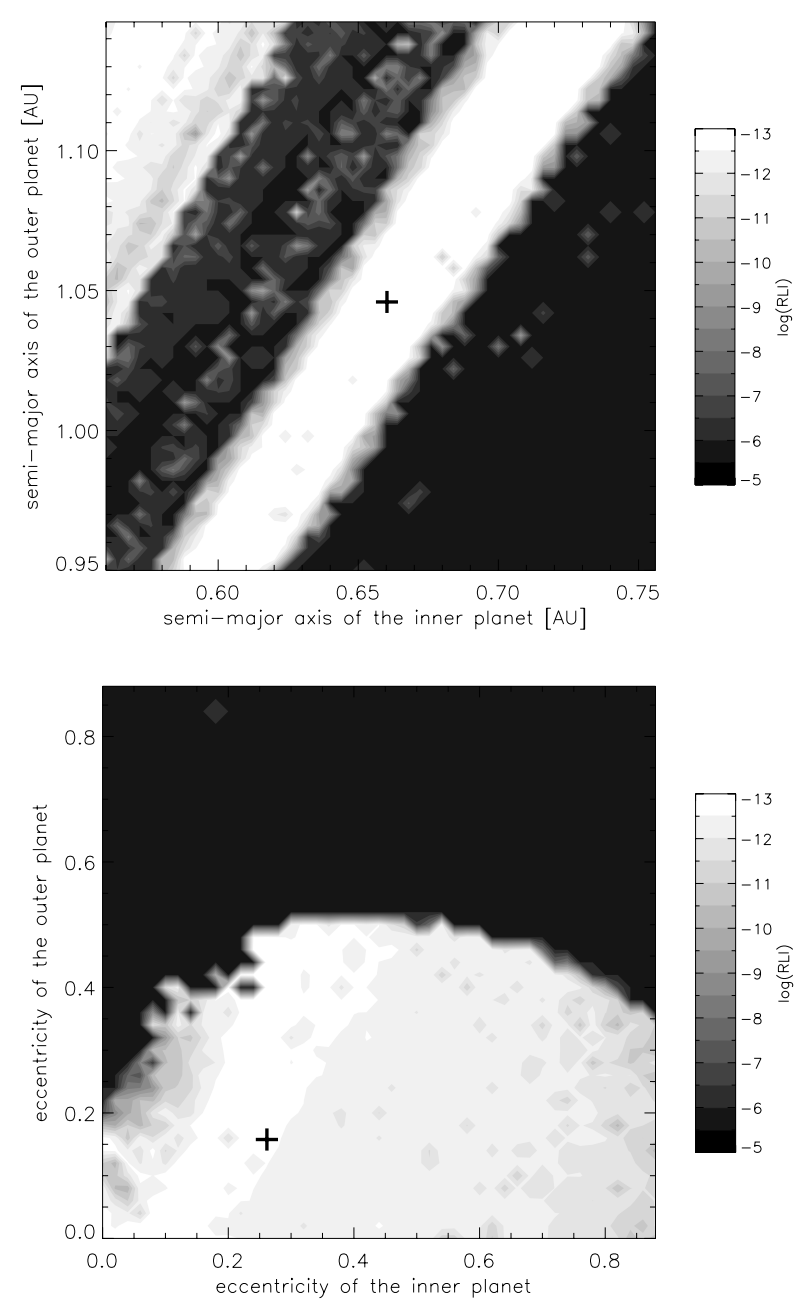

Fig. 3. Stability maps calculated using the orbital data provided by Fit 1 of Table 2. It can be seen from the parameter planes $a_{1}-a_{2}$ (top) and $e_{1}-e_{1}$ (bottom) that the orbital data of Fit 1 (marked by "+") are embedded well in the stable white region.

To investigate the stability of the newly derived orbital elements (shown in Table 2), we have computed (using the Relative Lyapunov indicator) a series of stability maps, similar to those shown in Fig. 2. We have found that in all cases the orbital elements are deeply embedded in the stable regions of the parameter planes $a_{1}-a_{2}, e_{1}-e_{2}, \varpi_{1}-\varpi_{2}$, and $M_{1}-M_{2}$. In Fig. 3 we show the stability structure of the parameter planes $a_{1}-a_{2}$ and $e_{1}-e_{2}$ in the case of Fit 1 . The semi-major axes and eccentricities corresponding to Fit 1 are marked with the symbol "+", and they lie almost in the middle of the stability region (white regions of the parameter planes). We note that at first sight the structure of the parameter planes $a_{1}-a_{2}$ corresponding to the 
Table 3. Properties of the dynamical orbit fits for HD 73526.

\begin{tabular}{cccc}
\hline \hline Fit No. & $\chi_{v}^{2}$ & rms $[\mathrm{m} / \mathrm{s}]$ & Offset velocity $[\mathrm{m} / \mathrm{s}]$ \\
\hline 1 & 1.81 & 8.36 & -46.67 \\
2 & 1.60 & 8.09 & -33.76 \\
3 & 1.87 & 8.4 & -49.54 \\
4 & 1.58 & 8.04 & -32.33 \\
\hline
\end{tabular}
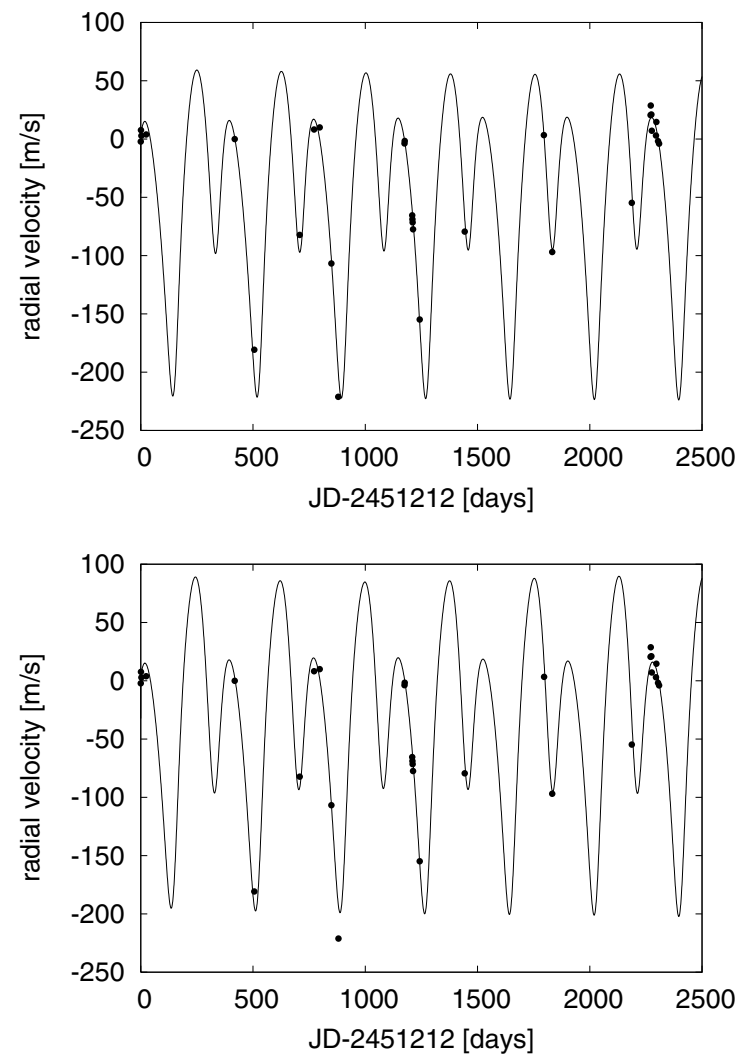

Fig. 4. Radial velocity curves of the star obtained by numerical integration using as initial conditions the orbital data given by Fit 1 (top panel) and Fit 4 (bottom banel) of Table 2. It can be seen that the calculated curves fit well to the measured radial velocity points (black dots).

original orbital data (shown in Fig. 2) and to the orbital data of Fit 1 (shown in Fig. 3) are similar. However, in Fig. 2 the stable regions are very narrow, the light regions (which may indicate stability) are mainly grey and have a fuzzy structure indicating the weakly chaotic character of the system. In Fig. 3 the light regions are white and they have a very homogeneous structure showing the ordered and therefore stable behavior of the system.

We have also checked by numerical integration whether the orbital data shown in Table 2 result in reliable radial velocity curves indicating the motion of the star. Two examples are shown in Fig. 4, corresponding to Fit 1 and Fit 4, respectively. We note that the radial velocity curves of Fit 1 and Fit 3 are very similar, while the radial velocity curves of Fit 2 and Fit 4 are very similar to the radial velocity curve given by Tinney et al. (2006) corresponding to their original dynamical orbit fit. In the case of Fit 1 and Fit 3 the mass of the inner giant planet is (slightly) smaller than the mass of the outer planet, which is also supported by the planetary migration scenario. According to full hydrodynamical simulations a cavity opens between the giant planets, and the inner planet orbits in a low density gaseous environment. The outer giant planet is still connected to the disk, thus it can accrete more
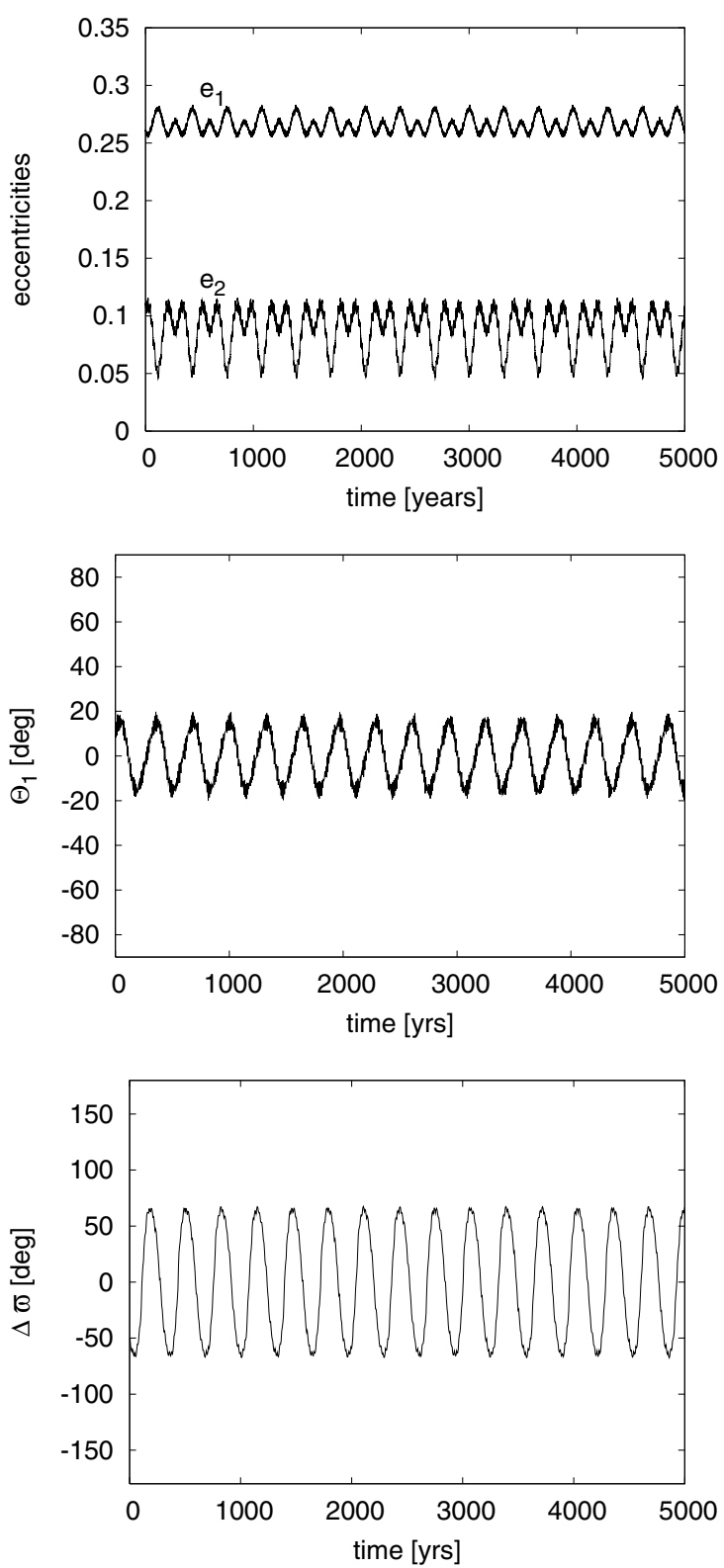

Fig. 5. The behavior of the eccentricities $e_{1}$ and $e_{2}$ (top), the resonant angles $\Theta_{1}$ (middle) and $\Delta \varpi$ (bottom) of the giant planets by using the initial orbital data given by Fit 3.

material, which may result in larger mass for it than for the inner planet (Kley et al. 2004).

The similarities between Fit 1 and Fit 3 or Fit 2 and Fit 4 can also be observed in the behavior of the eccentricities of the giant planets. The eccentricities in the cases of Fit 1 and 3 show moderate oscillations, while in the cases of Fit 2 and 4 they oscillate with very large amplitudes. One example for the behavior of the eccentricities is shown in the top of Fig. 5, corresponding to Fit 3. The other two (middle and bottom) panels of Fig. 5 show the behavior of the resonant angles $\Theta_{1}$ and $\Delta \varpi$.

In the following, when investigating the formation of the system HD 73526, we model the similar behavior provided by Fit 1 and Fit 3, in which case the outer planet is slightly more massive than the inner one, and the oscillations in the eccentricities are more moderate than in the cases of Fit 2 and Fit 4. 


\section{Formation of the system HD 73526 by smooth inward migration}

Since the present behavior of the planetary system around HD 73526 is very similar to the behavior of the system HD 128311, we investigate in this section the possibility that this behavior could be the result of a mixed formation scenario combining a smooth migration with a sudden perturbative effect as suggested by Sándor \& Kley (2006) in the case of HD 128311. We first model the evolution of the system by applying a slow inward migration. Having estimated the basic characteristics of the planetary migration, we study the formation of the system taking into account (i) a fast dispersal of the protoplanetary disk (resulting in the termination of the migration of the outer giant planet) and (ii) planet-planet scattering.

\subsection{A hydrodynamical model for $H D 73526$}

To study the general feasibility of forming a resonant system such as HD 73526 through migration of two embedded planets we performed full hydrodynamic evolutions where the accretion disk is treated in a flat two-dimensional approximation. The general setup and the applied numerical methods of the models are identical to those used in the detailed study of GJ 876 by Kley et al. (2005), see also Kley (1999). Here we restrict ourselves to a description of the particulars of our model for HD 73526, and concentrate on new features.

For the masses of the two planets we use $m_{1}=2.415 M_{\mathrm{J}}$ and $m_{2}=2.55 M_{\mathrm{J}}$ (see Fit 3 from Table 2), where $M_{\mathrm{J}}$ is the mass of Jupiter, and the planets are not allowed to accrete any mass during their evolution. The planetary orbits are initially circular at distances of $r_{1}=1$ and $r_{2}=2$ AU from the central star with mass $1.08 M_{\odot}$. The flat disk extends from of $r_{\min }=0.2$ to $r_{\max }=4.0 \mathrm{AU}$ with an initial surface density profile of $\Sigma(r)=\Sigma_{0} r^{-1 / 2}$. Here, the density at $r_{0}=1 \mathrm{AU}$ is normalized such that $\Sigma_{0} r_{0}^{2} / M_{\odot}=7.22 \times 10^{-4}$ which gives a total disk mass in the domain of about $0.024 M_{\odot}$. To make the initial evolution of the planets more realistic we superimpose initial density gaps on the unperturbed $r^{-1 / 2}$-profile (Kley 1999, 2000). The disk is driven by an $\alpha$-type viscosity with $\alpha=0.01$, and the temperature is specified using a fixed relative vertical thickness of $H / r=0.05$; no energy equation is solved. The smoothing length for the gravitational potential is $\epsilon=0.6 \mathrm{H}$, which gives approximately the correct values of the planetary migration rate when compared to the full three-dimensional case. For the torque cutoff, i.e. the region around the planet which is excluded in the calculation of the torques, we use $r_{\text {torq }}=0.6 R_{\text {Roche }}$. In the regular (non-resonant) evolution of the planets the exact values of $\epsilon$ and $r_{\text {torq }}$ should not influence the results too strongly due to the lack of material in the extended gap regions surrounding the planets. When the eccentricities of the planets increase during the evolution they periodically move through the disk at their peri- and apo-center and these numerical parameters may become more important. We have checked that our results do not depended on the exact values of $\epsilon$ and $r_{\text {torq }}$ as long as they are in the above specified range.

The computational domain from $r_{\min }$ to $r_{\max }$ is covered by 240 radial and 504 azimuthal gridcells where the radial spacing is logarithmic and the azimuthal equidistant. This gives roughly square gridcells throughout the whole domain. At the outer boundary we use damping boundary conditions where the disk profiles are relaxed to the initial profile (de Val-Borro et al. 2006). At the inner boundary we use a new type of boundary

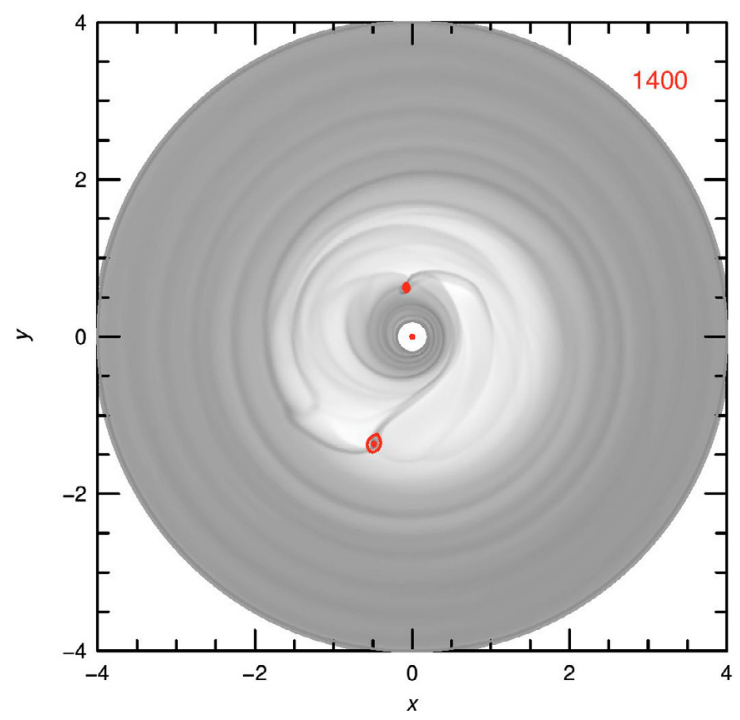

Fig. 6. Two dimensional density distribution of a protoplanetary accretion disk with two embedded planets after about 1400 years. From their initial positions ( $a_{1}=1.0$ and $\left.a_{2}=2.0 \mathrm{AU}\right)$ the planets have migrated inwards and, after being captured into a $2: 1$ resonance, have reached $a_{1}=0.85$ and $a_{2}=1.36 \mathrm{AU}$ with eccentricities $e_{1}=0.32$ and $e_{2}=0.08$. Within the inner planet, the inner disk has not been cleared away even after such a long evolution time due to a modified new inner boundary condition for the radial inflow velocity.

condition where we specify an outflow condition with an azimuthally averaged radial velocity which has a magnitude of

$v_{r}\left(r_{\min }\right)=-5 \frac{3}{2} \frac{v}{r_{\min }}$.

The typical viscous radial inflow velocity of accretion disks is $v_{\text {visc }}(r)=3 v /(2 r)$. We use here a 5 times higher value than $v_{\text {visc }}\left(r_{\text {min }}\right)$ to accomodate an increased clearing of the inner disk due to $i$ ) the disturbances of the embedded planets and $i$ ) the vicinity of the central star. Nevertheless, this boundary condition will prevent material leaving the inner disk too rapidly, which is typically observed in hydrodynamic evolutions with an embedded planet (Crida et al. 2007).

In Fig. 6 we show the distribution of the surface density $\Sigma(r, \varphi)$ of the protoplanetary accretion disk after about 1400 years. The inner planet is located at $x=-0.11, y=-0.61$ and the outer one at $x=-0.48, y=-1.37$. In contrast to previous models where the two planets orbit in an inner cavity of the disk without any inner disk (Kley et al. 2004), here the inner disk has not been cleared and is still present due to the more realistic inner boundary condition given in Eq. (1).

As a consequence the inner planet still is in contact with the inner disk which exerts gravitational torques on it. These tend to push the planet outward and will produce a damping of the eccentricities. In Fig. 7 we display the evolution of eccentricities and semi-major axis of the two planets. Initially, both planets orbit in a wide joint gap where the outer planet experiences only the torques of the outer disk and migrates inward, while the inner disk pushes the inner planet slightly outwards. Capture in the 2:1 mean motion resonance occurs at $400 \mathrm{yrs}$ after which the planets migrate jointly towards the star. The eccentricities increase strongly and reach equilibrium values at approximately 1100 yrs. In contrast additional full hydrodynamical test models that do not have an inner disk show a continued eccentricity increase in particular for the outer planet. We attribute this reduced magnitude of the eccentricities to the damping action of the 


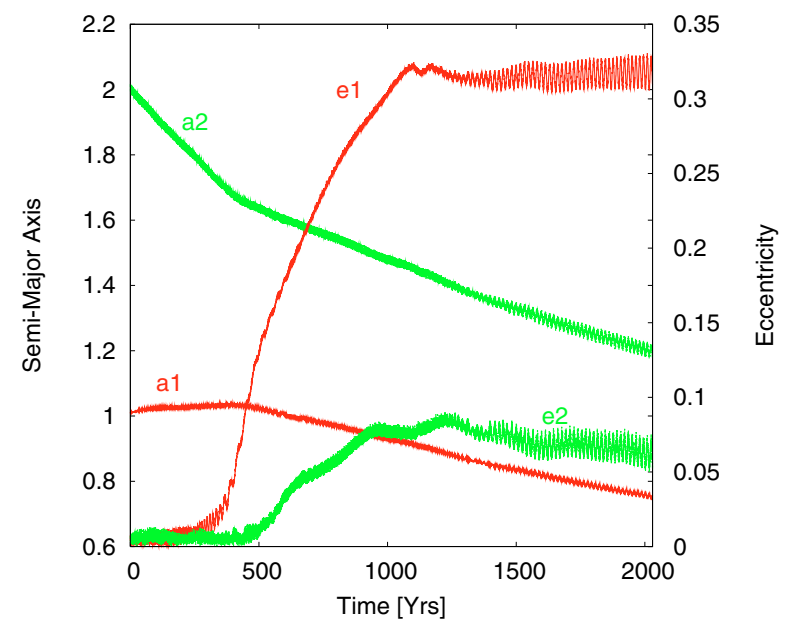

Fig. 7. The evolution of the semi-major axes and eccentricities of the two embedded planets for the full hydrodynamical model. The inner planet $\left(a_{1}, e_{1}\right)$ is indicated by the darker curves. After 400 yrs the outer planet captures the inner in a 2:1 mean motion resonance and they are coupled during their inward migration. The eccentricities increase rapidly after resonant capture and settle to equilibrium values for longer times.

inner disk. When the eccentricity of the inner planet increases it will periodically have to enter into the inner disk and the gravitational torques will tend to reduce its eccentricity. Equilibrium is then given by the eccentricity driving caused by the inward motion of the planets and the damping caused by the disk. After resonant capture the resonant angles $\Theta_{1}$ and $\Delta \varpi$ both librate with a libration amplitude of $\approx 35^{\circ}$ for $\Theta_{1}$ and $<10^{\circ}$ for $\Delta \varpi$. Hence, this hydrodynamical evolution displays an adiabatic migration process which results in apsidal corotation of the two planets, where the apsidal lines are always aligned.

In the long term the eccentricity of the outer planet $\left(e_{2}\right)$ remains at a level of 0.05 to 0.07 and does not decline any further. Additional test computations using different resolutions and boundary conditions confirm this trend. The hydrodynamical simulation shown represents an exploratory study to demonstrate that such a damping mechanism of the inner disk may have operated. Using this mechanism, it might be possible to accomodate a larger radial migration for the resonant planets than that found previously for the case of GJ 876 (Kley et al. 2005) because eccentricities will not be increased to very large values. This topic will be investigated in more detail in future work.

\subsection{Slow migration modeled by dissipative forces}

Hydrodynamical calculations modeling the migration of planets embedded in a gaseous protoplanetary disk require relatively long computational times. On the other hand, the damping effect of a protoplanetary disk to the orbital evolution of the embedded giant planets can also be modeled conveniently in the framework of the gravitational $N$-body problem by using well chosen nonconservative forces. These additional forces can be parametrized by the migration rate $\dot{a} / a$ and the eccentricity damping rate $\dot{e} / e$, or by the corresponding $e$-folding times $\tau_{a}$ and $\tau_{e}$ of the semimajor axis and eccentricity of the outer planet (see Lee \& Peale 2002; Beaugé et al. 2006). The relations between the damping rates and $e$-folding times are $\dot{a} / a=-1 / \tau_{a}$, and similarly for the eccentricities $\dot{e} / e=-1 / \tau_{e}$. We define the ratio between the $e$ folding times $K=\tau_{a} / \tau_{e}$ (or $\dot{e} / e=-K|\dot{a} / a|$ ) which according to
Lee \& Peale (2002) determines the final state of the system in the case of a sufficiently slow migration.

We recall that in the migration scenarios used up to now, mainly the damping of the outer giant planet has been taken into account. As we have mentioned previously, we have also supposed that beside the outer disk there is also an inner disk, and the planets migrates in a cavity between these disks. The presence of the inner disk accelerates the inner giant planet, thus forces it to migrate outward and also damps its eccentricity. This effect can also be modeled by using a (repelling) nonconservative force parametrized by $\dot{a} / a$ (having a positive sign for outward migration), and $K$ or $\dot{e} / e$.

In what follows, we present our results obtained in modeling the formation of the system HD 73526 by a slow migration process. We have studied three cases. In the first case, beside the damping effect of the outer disk, we have taken into account the effect of an inner disk. In the second case we have assumed that only the outer planet is affected by the outer disk and forced to migrate inward. In the third case we have assumed that after the resonant capture the protoplanetary disk disapears gradually, inhibiting the further increase of the inner planet's eccentricity. In this case only the outer planet has been forced to migrate inward. Our aim is to provide an evolution of the system which is in accordance with the eccentricity limits given by Fits $1-4$.

In order to compare the results of dissipative $N$-body calculations to those obtained by hydrodynamical simulations, at the beginning of the migration the giant planets move on circular orbits at distances $a_{1}=1 \mathrm{AU}$ and $a_{2}=2 \mathrm{AU}$ around a $M_{*}=1.08 M_{\odot}$ mass star. The masses of the giant planets have been fixed to $m_{1}=2.415 M_{\mathrm{J}}$ and $m_{2}=2.55 M_{\mathrm{J}}$, where $M_{\mathrm{J}}$ is the mass of Jupiter. For the inward migration of the outer giant planet we have applied $\tau_{a_{2}}=10^{4}$ years, while for the outward migration of the inner giant planet $\tau_{a_{1}}=-5 \times 10^{4}$ years. The ratio between the e-folding times in both cases was $K=10$. Comparing Fig. 7 with the top panel of Fig. 8, one can conclude that the presence of an inner disk can be modeled by a properly chosen dissipative force. The behavior of the resonant angles for this case is shown in the top panel of Fig. 9. Initially both $\theta_{1}$ and $\Delta \varpi$ deviate slightly from $0^{\circ}$ and tend finally to $0^{\circ} . N$-body calculations also confirm the results of the hydrodynamical simulations, that an inner disk can efficiently damp the eccentricity of an inner planet during a permanent migration.

Hydrodynamical simulations for HD 73526 also show that the lack of an inner disk results in a continuous increase of the eccentricity of the inner giant planet. In what follows, we will study which value of the ratio $K$ is necessary to stop the increase of the inner planet's eccentricity when no inner disk is considered (It is known from the hydrodynamical simulations that $K$ typically lies between 1-10).

We have applied a damping of the semi-major axis of the outer planet with an $e$-folding time $\tau_{a_{2}}=10^{4}$ year using different $K$ values ( $K=10,15$, and 20). The time evolution of the semi-major axes and the eccentricities are shown in the middle panel of Fig. 8, while the behavior of the resonant angles $\theta_{1}$ and $\Delta \varpi$ (with $K=10$ ) is displayed in the middle panel of Fig. 9. Studying the behavior of the eccentricities for the different ratios $K$ one can see that during the migration process the eccentricity of the outer planet is damped sufficiently, while the eccentricity of the inner planet is slightly increasing. If the migration is terminated when the semi-major axes reach their actual values (at $t \approx 1.3 \times 10^{4}$ year), we need $K \sim 15$ not to exceed the observed eccentricity of the inner planet. (The upper limit for the eccentricity of the inner planet is around $e_{1 \max } \approx 0.3$, as it has been shown by our numerical integrations based on the orbital 

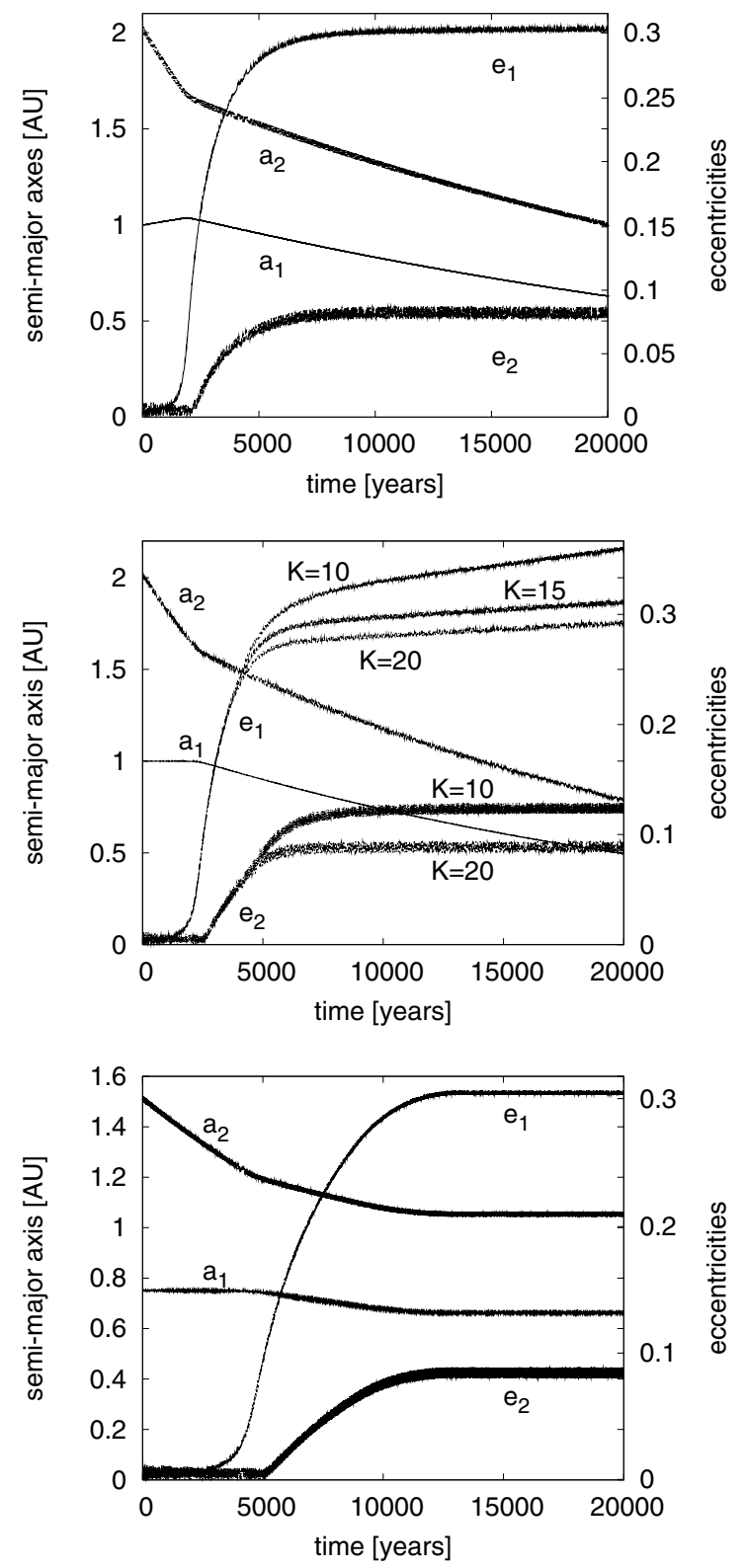

Fig. 8. Evolution of the semi-major axes and the eccentricities of the giant planets during different migration scenarios: (i) the actions of an outer and an inner disk are modeled, $\tau_{a_{2}}=10^{4}$ years, $\tau_{a_{1}}=-5 \times$ $10^{4}$ years and $K_{1}=K_{2}=10$ (top); (ii) only the damping effect of an outer disk is modeled, $\tau_{a_{2}}=10^{4}$ years and $K=10,15$, and 20 (middle); and (iii) the resonant capture takes place right before the disk's dispersal, obeying a linear reduction law between $9 \times 10^{3}$ and $1.3 \times$ $10^{4}$ years, $\tau_{a_{2}}=2 \times 10^{4}$ years, and $K=10$ (bottom).

data of Fits 1-4.) On the other hand, from hydrodynamical simulations we know that $K \sim 1-10$, thus although the above values $K=15$ seem to be slightly high, it may be acceptable for thick disks. But the $K$ value can be reduced to $K_{1}=K_{2}=10$ by the presence of an inner disk as shown above by the hydrodynamical simulations. The evolution of the resonant angles $\Theta_{1}$ and $\Delta \varpi$ (for $K=10$ ) are shown in the middle a panel of Fig. 9. After the resonant capture, $\Theta_{1}$ and $\Delta \varpi$ deviate from $0^{\circ}$, and having reached their extrema $\left(\sim 20^{\circ}\right.$ for $\Theta_{1}$ and $\sim 50^{\circ}$ for $\left.\Delta \varpi\right)$, they tend to $0^{\circ}$ very slowly. The reason for this behavior might be that the mass ratio between the giant planets $\left(m_{1} / m_{2}=0.947\right)$ lies very close to the critical mass ratio $\left(m_{1} / m_{2}\right)_{\text {crit }}=0.95$ given by Lee (2004). If $m_{1} / m_{2}>\left(m_{1} / m_{2}\right)_{\text {crit }}$, the resonant system evolves
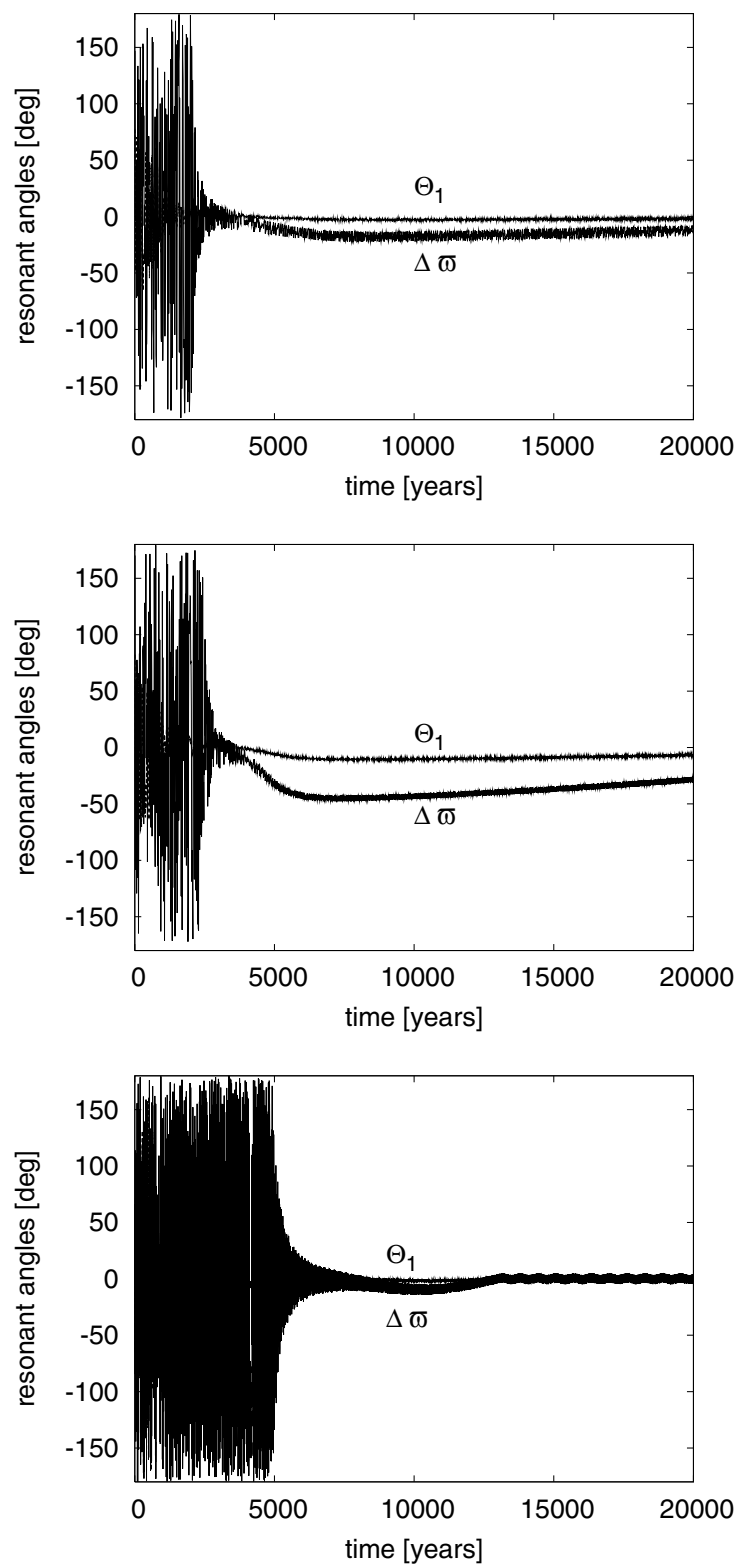

Fig. 9. Evolution of the resonant angles $\Theta_{1}$ and $\Delta \varpi$ during the migration scenarios corresponding those displayed in Fig. 8.

temporarily through an asymmetric apsidal configuration. We note that the deviation of the angles $\Theta_{1}$ and $\Delta \varpi$ is the largest in the the above case when only the outer giant planet is damped by an outer disk.

It is also clear that the migration of the giant planets should terminate when they reach their actual positions. If the termination of the migration is a slow process the system reaches a state very close to a periodic solution of the corresponding threebody problem studied by Psychoyos \& Hadjidemetriou (2005), for instance.

In order to avoid the use of the relatively high values for $K \sim 15-20$, we have also studied the possibility when the resonant capture between the giant planets takes place just before the dispersal of the protoplanetary disk. We recall that this scenario (proposed by Kley et al. 2005) might have occurred in the case of GJ 876, if there is no strong damping mechanism acting on the eccentricities. We have supposed that prior to the resonant capture the giant planets migrated separately to their orbits having $a_{1}=0.75 \mathrm{AU}$, and $a_{2}=1.5 \mathrm{AU}$. Then the outer planet 
migrated inward with an $e$-folding time of its semi-major axis $\tau_{a}=2 \times 10^{4} \mathrm{yr}$ with $K=10$, and captured the inner planet into the 2:1 resonance, see the bottom panel of Fig. 8. The resonant capture takes place around $t \approx 5 \times 10^{3}$ years, the eccentricity of the inner planet grows rapidly, but before exceeding the limit $e_{1 \max }=0.3$, the protoplanetary disk has already disappeared and thus the migration is terminated. The disk dispersal happens between $9 \times 10^{3}-1.3 \times 10^{4}$ years, obeying a linear reduction law. During this simulation the final value of the inner planet's eccentricity remained around the critical value $e_{1} \leq 0.3$, however the ratio of the $e$-folding times was only $K=10$.

\section{Formation of the system HD 73526 by mixed evolutionary scenarios}

In the previous section we presented three possible migration scenarios that can push the giant planets deep into the 2:1 resonance not contradicting to the observed upper limits of the eccentricities $\left(e_{1} \sim 0.3, e_{2} \sim 0.1\right)$. On the other hand, as our dynamic orbit fits (Fit 1-4) in Table 2 show, the eccentricities of the giant planets and the resonant angles oscillate with considerable amplitude. This behavior is similar to that observed in the case of the system HD 128311, thus in what follows, we model the formation and early evolution of the system HD 73526 by using mixed evolutionary scenarios combining inward migration and the sudden perturbative events mentioned in Sándor \& Kley (2006).

\subsection{Sudden termination of migration}

Recent Spitzer observations of young stars show that the inner part of the protoplanetary disk may be emptied due to photoevaporation induced by the central star (see D'Alessio et al. 2005; Calvet et al. 2005). Thus when approaching the inner rim of such a disk, the inward migration can be stopped rapidly (Masset et al. 2006). If the inner rim is located at a given radius of the disk (e.g. it is stationary) the migration cannot be maintained because in this case the the outer disk, which is the driving agent of the migration, cannot follow the planet on its inward drift.

Based on our experience in modelling the formation of the system HD 128311, we investigated the above case when the termination of the migration (due to the presence of a stationary inner rim) happens on a very short timescale, here $\Delta t_{\text {dis }}=50$ years. In this case we apply a faster migration $\tau_{a}=4 \times 10^{3}$ years and $K=15$. The behavior of the eccentricities and the resonant angles $\Theta_{1}$ and $\Delta \varpi$ is shown in Fig. 10. This fast termination of the migration can result in a behavior of the eccentricities and the resonant angles similar to the observed case shown in Fig. 5.

The reason for this behaviour is that at the beginning of the migration process, the resonant angles deviate from $0^{\circ}$, reach their extrema, and tend very slowly to $0^{\circ}$ (see also the middle panel of Fig. 9). If the termination of the migration occurs when the resonant angles still deviate from $0^{\circ}$, they begin to oscillate (with an amplitude equal to their values at the end of the disk's dispersal) around their equilibrium (which in this case is $0^{\circ}$ ). This scenario works also in the cases of slower migration rates; however in the case of a faster migration the deviation of the resonant angles from $0^{\circ}$ is larger, and hence the amplitude of the oscillations in $e$, is noticeably higher.

We note that the above fast migration of protoplanets may not be likely in protoplanetary disks. In thick disks fast migration may occur, but in this case it is not easy to find relevant physical processes leading to the sudden termination of migration.
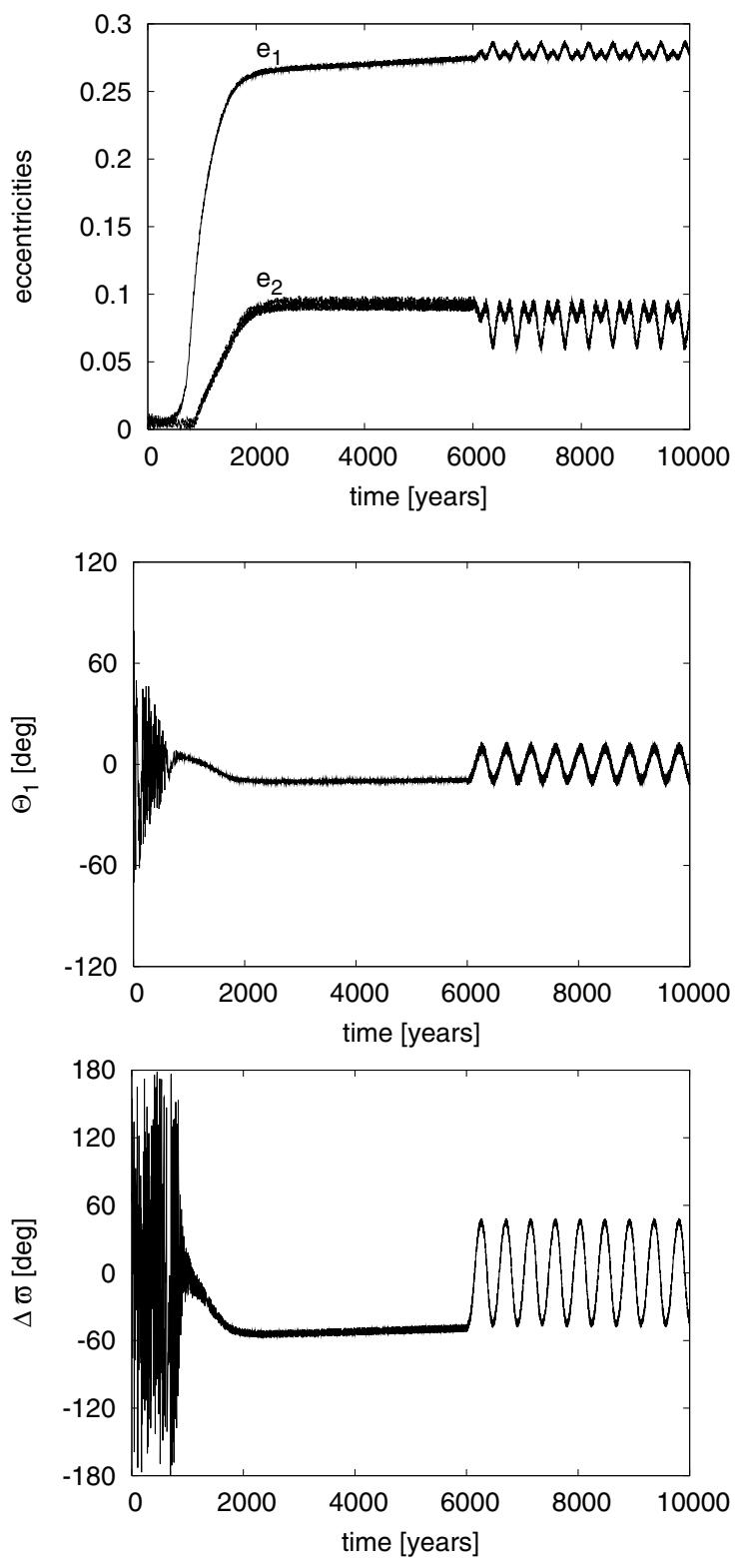

Fig. 10. Top: behavior of the eccentricities of the giant planets during an inward migration followed a sudden dispersal of the protoplanetary disk, $\tau_{a_{2}}=4 \times 10^{3}$ and $K=15$. The giant planets orbit initially at $a_{1}=1 \mathrm{AU}, a_{2}=2 \mathrm{AU}$, and the migration is stopped between 6000 and $6050 \mathrm{yrs}$, applying a linear disk dispersal law. The fast termination of the migration results in oscillations of the eccentricities of the planets, very similar to that shown in Fig. 4. Middle and bottom: behavior of the resonant angles $\Theta_{1}$ and $\Delta \varpi$ corresponding to the above scenario. After the fast termination of the migration the resonant angles also behave very similarly to the case displayed in Fig. 4.

However, the outcome of the sudden stop of migration characterized by the above parameters yields results in good agreement with the behavior of the system using as initial conditions the data of Fit 3.

\subsection{Scattering with a small mass inner planet}

The behavior of the eccentricities of the giant planets around HD 73526 is very similar to that observed in the system HD 128311 and $v$ Andromedae. In the case of $v$ Andromedae, Ford et al. (2005) proposed that such a behavior is most likely 
the result of a planet-planet scattering event. Studying the system HD 128311, Sándor \& Kley (2006) demonstrated that the observed behavior of that system may be the consequence of planet-planet scattering as well. Tinney et al. (2006) have also suggested that the dynamical behavior of the system around HD 73526 might be the result of a dynamical scattering event. In what follows, we investigate whether the present behavior of HD 73526 can be modeled by such an effect.

We have assumed that in addition to the giant planets, already being in a 2:1 resonance and migrating towards the host star, a small mass planet $\left(\sim 10 M_{\oplus}\right)$ is also orbiting close to the host star in a quasi circular orbit. (This assumption could be realistic, as the discovery of a $7.5 M_{\oplus}$ planet around GJ 876 shows, Rivera et al. 2005) Initially, the giant planets are far enough from the small mass planet that they do not significantly influence its motion. However, as the giant planets migrate inward approaching their present positions, they perturb the motion of the small planet. As our numerical experiments show, at the corresponding ratio of the semi-major axes the inner giant planet captures the small mass planet into a 3:1 resonance. Once the capture into the 3:1 resonance happens, two different scenarios can occur: (i) the eccentricity of the small mass planet initially grows, then oscillates around the eccentricity of the inner giant planet, (ii) the eccentricity of the small mass planet grows during the whole migration process, which can result in a possible close encounter between the inner small mass and the giant planet.

In the case (i) the resonant angles of the $3: 1$ resonance $\theta_{1}=3 \lambda_{1}-\lambda_{\text {inn }}-\varpi_{1}-\varpi_{\text {inn }}, \theta_{2}=3 \lambda_{1}-\lambda_{\text {inn }}-2 \varpi_{1}$, and $\theta_{3}=3 \lambda_{1}-\lambda_{\text {inn }}-2 \varpi_{\text {inn }}$ librate around $\sim 40^{\circ}, \sim 150^{\circ}$, and $\sim 290^{\circ}$, respectively, while $\Delta \varpi^{*}=\varpi_{1}-\varpi_{\text {inn }}$ also librates around $\sim-110^{\circ}$ showing that the orbits of the inner giant and of the small mass planet are in an asymmetric apsidal corotation. Here the index " 1 " refers to the inner giant planet, the index "inn" to the small mass inner planet. This resonant configuration protects the small mass inner planet from the close encounter (making planet-planet scattering impossible), and stabilizes its orbit throughout migration. The behavior of the eccentricities of the planets and the resonant angle $\theta_{1}$ (for the 3:1 resonance), $\Delta \varpi^{*}$ are shown in Fig. 11.

In the case (ii) the inner giant planet also captures the small mass planet into a 3:1 resonance, however, in this case only $\theta_{1}$ librates around $0^{\circ}$ (see the bottom panel of Fig. 12), the other resonant angles are circulating, thus the orbits of the inner giant and the small mass planets are not in apsidal corotation. During the migration, the eccentricity of the small mass inner planet grows (shown in the top panel of Fig. 12) resulting in progressively more elongated orbits. Thus eventually the orbit of the small mass planet will cross the orbit of the inner giant planet and a close encounter can happen between the planets. After the close encounter the small planet is either ejected from the system or pushed into an orbit distant from the host star.

Having performed several numerical simulations, we have found that the outcome of the resonant capture described in the cases (i) and (ii) depends mainly on the initial positions of the inner giant and small mass planet, and it is not very sensitive to the mass of the small inner planet (ranging between 0.03 and $0.04 M_{\text {Jup }}$ ). It is very probable that prior to its secular increase, $e_{\text {inn }}$ goes through a sudden change in the family of corotation described and also analysed recently for the 3:1 resonance by Michtchenko et al. (2006). Thus a detailed analysis would be useful to study the mechanism and the probability of resonant capture into the 3:1 resonance leading to either a protective behavior or a close encounter with the inner giant planet and a possible ejection of the small mass planet. However, at the present
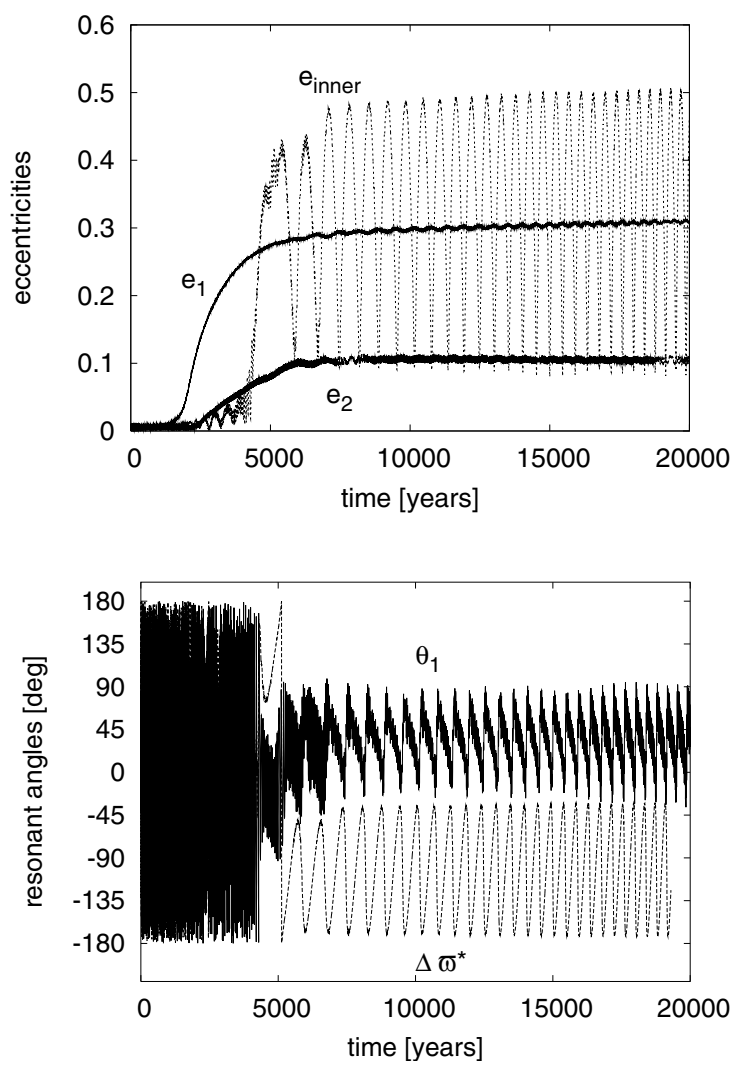

Fig. 11. Top: the evolution of the eccentricities of the giant and the small mass planets during planetary migration when the inner giant planet captures the small mass inner planet into a protective 3:1 resonance. This resonant configuration inhibits the increase of the small mass inner planet's eccentricity thus the system remains stable troughout the migration process. Bottom: the behavior of the resonant angles $\theta_{1}$ and $\Delta \varpi^{*}$ shows that beside the 3:1 resonance, the orbits of the inner giant and the small mass planet also are in an asymmetric apsidal corotation.

stage of our investigations we restrict ourselves to demonstrating the mechanism that leads to the increase of the eccentricity of the small mass inner planet and to a planet-planet scattering event.

In our numerical experiments we varied the mass of the small planet between 0.03 and $0.04 M_{\text {Jup }}$ and the migration timescale as well. We have performed several simulations, and have found that once scattering happens, in the majority of the cases the system remains captured into the 2:1 resonance. However, in some cases we have observed the complete destruction of the resonance, or even escape of the giant planets. If the scattering takes place well before the disk dispersal process, the large oscillations in the eccentricities may be smoothed out due to the damping of the disk acting on the eccentricity of the outer giant planet. If the scattering happens during or after the disk dispersal, the oscillations of the eccentricities are preserved, and the system reaches its presently observed state. We have found that in order to observe the continuous increase of the small mass inner planet's eccentricity the migration of the giant planets should take a sufficiently long time.

The question may arise whether it is necessary to assume a temporal synchronization between the termination of the migration and the scattering event. We have performed several numerical investigations and found in many cases that the inward migrating giant planets represent a perturbation strong enough to make the behaviour of the small mass inner planet unstable. However, the small planet survived for longer times, and 

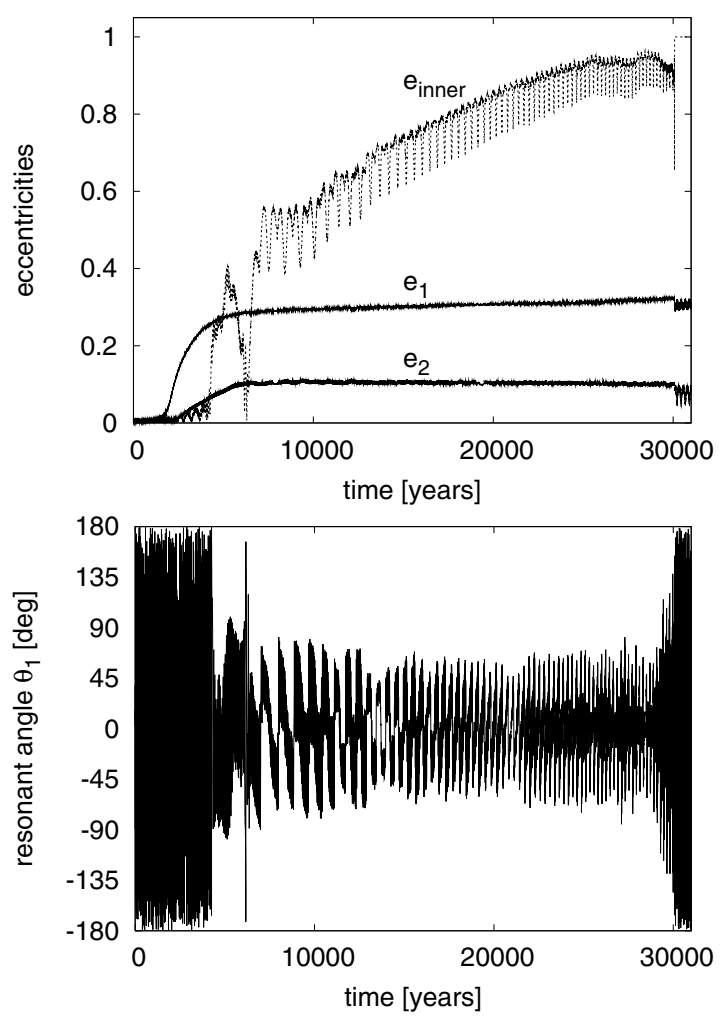

Fig. 12. Top: the evolution of the eccentricities of the giant and the small mass planets during planetary migration when the small mass planet is also captured into a 3:1 resonance by the inner giant planet. In this case the resonant configuration is no longer protective the eccentricity of the small mass planets increases during the migration and its orbit can cross the orbit of the inner giant planet. Bottom: the behavior of the resonant angle $\theta_{1}$ during the migration process. We note that the other resonant angles (of the 3:1 resonance) are circulating, thus the orbits of the inner giant and the small mass inner planet are not in apsidal corotation.

the close encounter happened when the migration had terminated. On the other hand there can also be a natural temporal synchronization. The migration of the giant planet should terminate when they reach their presently observed position. If a small mass planet orbits at a distance from the central star, needed for the resonant capture, its orbit may easily be captured into a higher order resonance, and therefore its eccentricity increased. This will allow a close encounter with the inner giant planet, and finally, its ejection from the system.

In Fig. 13, which is a magnification of Fig. 12, we show the typical behavior of the eccentricities of the giant planets, and $\Delta \varpi$ during a migration process before and after a planet-planet scattering. The giant planets are started on distant orbits having initial semi-major axes $a_{1}=2 \mathrm{AU}$ and $a_{2}=4 \mathrm{AU}$. The $e$-folding time of the outer giant's semi-major axis is $\tau_{a_{2}}=10^{4}$ years and $K=15$.

The mass of the inner small planet is $M_{\text {inn }}=0.027 M_{\text {Jup }}$, and it orbits at $a_{\mathrm{inn}}=0.9 \mathrm{AU}$ from the host star in a nearly circular orbit. The scattering occurs around $t_{\mathrm{sc}}=3 \times 10^{4}$ years. We applied a linear dispersal process of the protoplanetary disk taking place between $2.9 \times 10^{4}$ and $3.1 \times 10^{4}$ years. Thus the oscillations in the eccentricities induced by the scattering event are damped no longer. Since the giant planets are still in a (protective) 2:1 resonance their stability is preserved for the whole life-time of the system. We note that in contrary to the case HD 128311, during modeling the formation of HD 73526 we have not observed the
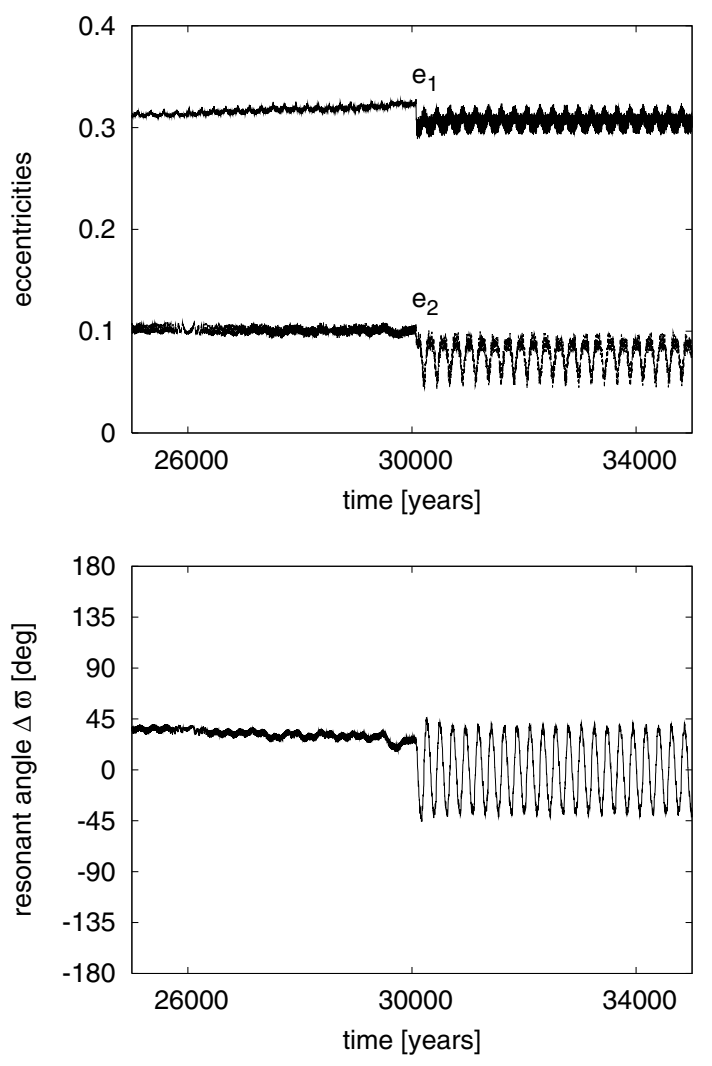

Fig. 13. Top: the behavior of the eccentricities of the giant planets during a planetary migration process before and after a planet-planet scattering event taking place around $t_{\mathrm{sc}} \approx 3 \times 10^{4}$ years. Bottom: the behavior of $\Delta \varpi$. The behavior of the eccentricities and $\Delta \varpi$ is very similar of those of Fit 3.

breaking of the apsidal corotation. It is highly probable that due to the large planetary masses and relatively small semi-major axes, the system is more fragile than HD 128311, and a sufficiently strong perturbation which could lead to the breaking of the apsidal corotation may destroy the whole system. However, after the scattering event the resonant angle $\Delta \varpi$ shows large oscillations around $0^{\circ}$.

We note that in the above simulations (gravitational threebody problem with dissipative forces) the small mass inner planet has not been affected by the inner disk. In this case we used a model without an inner disk, which may also be realistic in some cases. However, the damping ratio $K=15$ is a bit high; smaller $K$ would result in higher eccentricity of the inner giant planet. As we have already demonstrated in 4.1, the presence of an inner disk can effectively damp the eccentricity of the inner giant planet. In order to investigate the effect of the inner disk on the small mass planet we have also performed full hydrodynamical simulations using the same initial parameters as described in 4.1 , but in addition to the giant planets, a $M_{\mathrm{inn}}=10 M_{\oplus}$ inner planet has been embedded in the inner disk and started from $0.4 \mathrm{AU}$. According to the theoretical expectations, its migration rate (being in the linear regime with no gap opening) is smaller than that of a more massive Jupiter-like planet. In this full hydrodynamical case the small mass planet has been captured into a 4:1 resonance resulting in an increase of its eccentricity. In Fig. 14 we display the time evolution of the orbital elements of the three planets. At $t=400$ orbital periods (of the inner giant planet) the two massive planets capture each other in a $2: 1$ resonance, slowing down the inward migration which nevertheless 

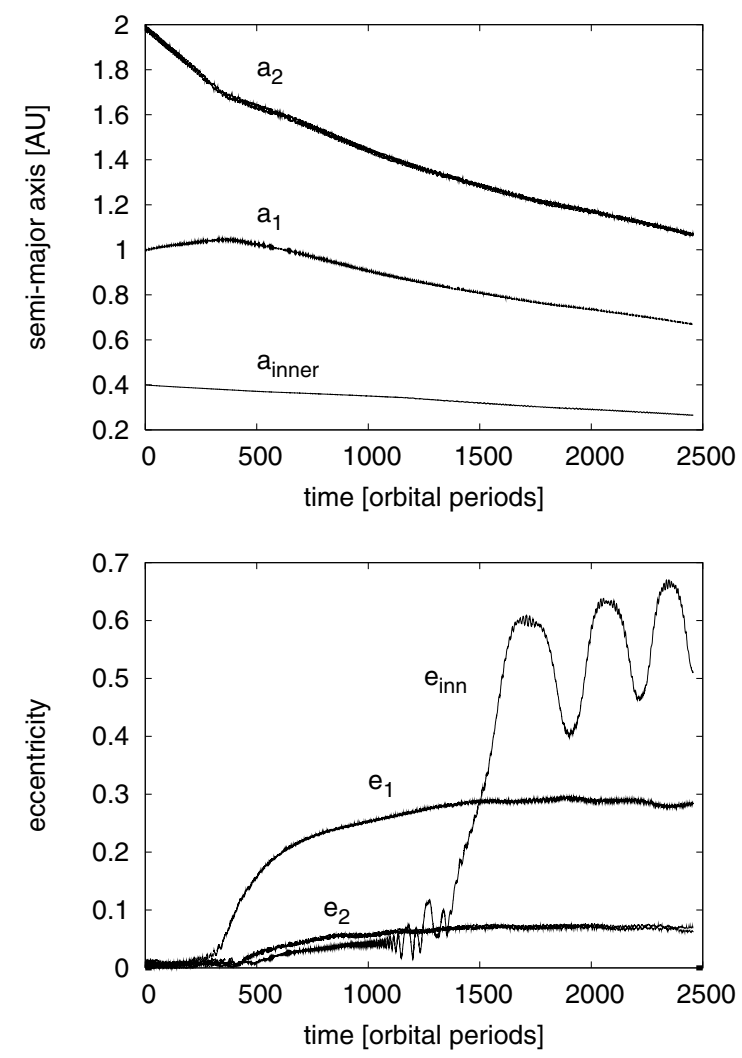

Fig. 14. Full hydrodynamical evolution of two embedded massive planets and an small additional small mass inner planet. Top: the behavior of the semimajor axis of the three planets. Bottom: the behavior of the eccentricities.

remains faster than that of the inner planet which is consequently captured at a later time. Due to the very long computational time, we have not followed the complete evolution of the small mass inner planet. On the other hand it is very probably, that the increase of its eccentricity will result in a scattering with the inner giant planet, and finally, ejection from the system. Similarly to the eccentricity damping mechanism, the final behavior of the inner small mass planet will be the subject of a more detailed analysis investigating inner disks with different physical properties. However, our results show that even in the presence of an inner disk the eccentricity excitation mechanism of the small mass inner planet (through resonant capture) works well, and enables scattering between the small mass and the inner giant planet.

\section{Conclusion}

We have investigated different evolutionary scenarios which may have led to the observed behavior of the resonant system HD 73526. According to our numerical integration, the original orbit fit provided by Tinney et al. (2006) results in weakly chaotic motion. Thus, we have derived four sets of orbital elements, which fit very well to the observed radial velocity measurements, and also provide, through a protective $2: 1$ resonance, stable motion for the giant planets during the whole life-time of the system. Two of these fits yield very large variations of the eccentricities of the giant planets; in one case the giant planets are not even in apsidal resonance. In the case of the other two fits the variations of the eccentricities are more moderate, and beside the mean motion resonance, the giant planets are in apsidal corotation.
Since the behavior of the giant planet's eccentricities in the system HD 128311 is very similar to those in HD 73526, we have investigated whether this system may also be the result of a mixed formation scenario incorporating a slow migration followed by a perturbative event. Similarly to the formation of the system HD 128311, we have assumed that the two giant planets have been formed far from their hosting star, and migrated inward due to a planet-disk gravitational interaction. During this migration the planets have been locked in a protective 2:1 resonance.

Performing full hydrodynamical simulations of two embedded giant planets in a protoplanetary disk we have found that the presence of an inner disk can efficiently damp the eccentricity of the inner giant planet resulting in eccentricity values in good agreement with the observations. This mechanism has not been investigated yet, and may offer a real possibility to avoid the use of very high values of the ratio $K$, for example in modeling the resonant system around GJ 876 . We have additionally found that the effect of an inner disk can be modeled very conveniently by using non-conservative forces. We have investigated three convergent migration scenarios that can lead the system to the deep 2:1 resonance, which are all physically realistic.

Similarly to HD 128311 we have assumed that the system HD 73526 suffered a sudden perturbation near the end of the migration of the giant planets. This perturbation could either be the quick termination of the migration, which may be induced by an inner rim of the disk and an empty region inside of it, as indicated by some observations of young stars, or a planet-planet scattering event. We have analyzed the results of an encounter of one of the giant planets with a relatively small $\sim 10 M_{\oplus}$ planet, which orbits around the central star at a closer distance $(a<$ $1 \mathrm{AU}$ ), and which is ejected from the system, or thrown to a very distant orbit after the scattering event.

Investigating the effect of the disk's sudden dispersal, we have found that only a very short disk dispersal time $\Delta t_{\text {disp }}=$ 50 years will induce the observed oscillations in the eccentricities and in the resonant angles. The reason for this behavior is that during the migration of the giant planets the system tends to an asymmetric apsidal corotation with $\Delta \varpi \neq 0^{\circ}$. At the moment of the sudden termination of the migration $\Delta \varpi \approx 50^{\circ}$, and $\Delta \varpi$ will continue to librate with this amplitude around its equilibrium $\left(0^{\circ}\right)$. If $\Theta_{1}$ is also different from $0^{\circ}$, it will also librate around $0^{\circ}$ after the scattering event. We note however that in order to obtain the present behavior of the system we need relatively fast migration of the giant planets $\tau_{a_{2}}=4 \times 10^{3}$ years and $K=15$.

Studying the planet-planet scattering event we have found that the inner giant planet frequently captures a small mass inner planet in a higher order resonance (here $3: 1$ or $4: 1$ ). Then the further evolution of the system depends on whether the orbits of the giant and the small mass planet are in apsidal corotation after the resonant capture. The apsidal corotation protects the orbit of the small mass planet from the close encounter with the inner giant planet, thus inhibiting a planet-planet scattering event. The lack of apsidal corotation results in a continuous increase of the small mass planet's eccentricity. Thus its orbit crosses the orbit of the giant planet and a close encounter can take place between them. Afterwards, if the system survives that perturbative event, the eccentricities of the giant planets behave qualitatively similarly to those of our Fit 3, in agreement with the observed radial velocity observations of HD 73536.

We can conclude that based on radial velocity measurements to date, it is probable that during the formation and early evolution of the systems HD 73526 and HD 128311 the 
adiabatic migration, which brought the systems into a protective 2:1 resonance, was followed by a sudden perturbative event. This perturbative event could be either the fast dispersal of the circumstellar disk or planet-planet scattering. Thus the dynamics of the system HD 73526 confirm that planetary migration followed by sudden perturbations (e.g. planet-planet scattering) play an important role in shaping the formation and early dynamics of resonant planetary systems.

Acknowledgements. We thank Prof. C. Beaugé for his careful reading of the manuscript and his useful comments and suggestions. This work has been supported by the Hungarian Scientific Research Fund (OTKA) under the grant PD48424 and by the German Research Foundation (DFG) under grant 436 UNG 17/1/07.

\section{References}

Beaugé, C., Michtchenko, T. A., \& Ferraz-Mello, S. 2006, MNRAS, 365, 1160 Bois, E., Kiseleva-Eggleton, L., Rambaux, N., \& Pilat-Lohinger, E. 2003, ApJ, 598, 1312

Calvet, N., D'Alessio, P., Watson, D. M., et al. 2005, ApJ, 630, L185
Crida, A., Morbidelli, A., \& Masset, F. 2007, A\&A, 461, 1173 D’Alessio, P., Hartmann, L., Calvet, N., et al. 2005, ApJ, 621, 461 de Val-Borro, M., Edgar, R. G., Artymowicz, P., et al. 2006, MNRAS, 370, 529 Ford, E. B., Lystad, V., \& Rasio, F. A. 2005, Nature, 434, 873

Hayashi, C. 1981, Progr. Theor. Phys. Suppl., 70, 35

Kley, W. 1999, MNRAS, 303, 696

Kley, W. 2000, MNRAS, 313, L47

Kley, W., Peitz, J., \& Bryden, G. 2004, A\&A, 414, 735

Kley, W., Lee, M. H., Murray, N., \& Peale, S. J. 2005, A\&A, 437, 727

Lee, M. H. 2004, ApJ, 611, 517

Lee, M. H., \& Peale, S. J. 2002, ApJ, 567, 596

Masset, F. S., Morbidelli, A., Crida, A., \& Ferreira, J. 2006, ApJ, 642, 478

Michtchenko, T. A., Beaugé, C., \& Ferraz-Mello, S. 2006, Celest. Mech. Dyn. Astron., 94, 411

Perryman, M. A. C. 2000, Rep. Progr. Phys., 63, 1209

Pollack, J. B. 1984, ARA\&A, 22, 389

Psychoyos, D., \& Hadjidemetriou, J. D. 2005, Celest. Mech. Dyn. Astron., 92, 135

Rivera, E. J., Lissauer, J. J., Butler, R. P., et al. 2005, ApJ, 634, 625

Sándor, Z., \& Kley, W. 2006, A\&A, 451, L31

Sándor, Z., Érdi, B., Széll, A., \& Funk, B. 2004, Celest. Mech. Dyn. Astron., 90, 127

Tinney, C. G., Butler, R. P., Marcy, G. W., et al. 2006, ApJ, 647, 594

Vogt, S. S., Butler, R. P., Marcy, G. W., et al. 2005, ApJ, 632, 638 